\title{
Vitamin D as an adjuvant in melanoma therapy
}
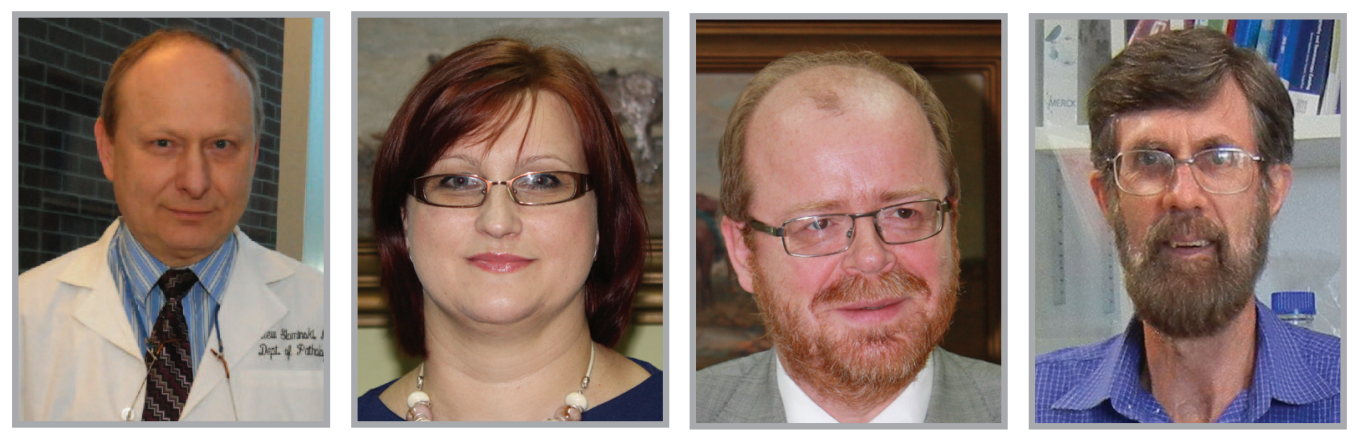

\section{Melanoma Management}

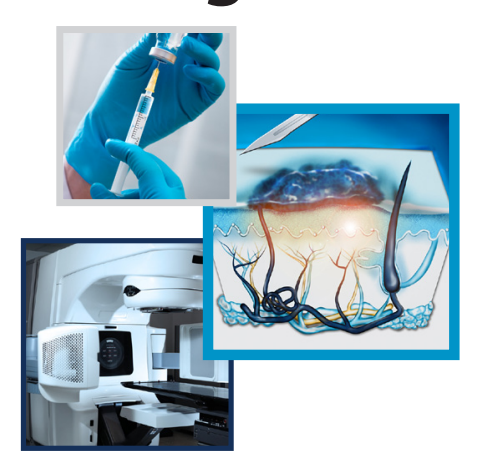

Andrzej T Slominski*,1,2, Anna Brozyna ${ }^{3}$, Wojciech Jozwicki ${ }^{3}$

\& Robert C Tuckey ${ }^{4}$

Metastatic melanoma: a formidable challenge for clinicians \& researchers

Recent advances in our understanding of the principles underlying melanoma behavior $[1,2]$ have led to an explosion in the strategies proposed to treat cutaneous metastatic melanomas [1-3]. In effect, new molecules and treatment modalities are being developed [1-4] to treat this devastating disease. Unfortunately, these strategies only retain efficacy for a few months before the disease relapses, plus the drugs involved have toxic side effects and are very expensive [5], so the only reliable therapy is proper surgical excision when the disease is localized to the skin.

Therefore, the management of metastatic melanoma represents a significant challenge for both clinicians and patients, and there is an urgent need to clarify the mechanisms responsible for resistance to therapy against metastatic melanoma [5]. These challenges undoubtedly will be addressed by basic and clinical melanoma researchers for years to come. However, to help patients with these devastating diseases we need immediate, nontoxic and economical adjuvant strategies for improvement of patient survival, with relatively limited side effects. These requirements can be met by the application of natural products and their derivatives in an educated fashion, to not only restrict melanoma growth and progression but also to restore the body's homeostatic capabilities to control tumor behavior [5]. Examples of such compounds are vitamin $\mathrm{D}$ that can be activated in the body by the classical pathway to $1 \alpha, 25$-dihydroxyvitamin $\mathrm{D}$ $\left(1,25(\mathrm{OH})_{2} \mathrm{D}\right)[6,7]$, and newly discovered forms of vitamin $\mathrm{D}[8,9]$ that show antiproliferative, anti-inflammatory and anticancer properties [10].

'Department of Pathology \& Laboratory Medicine, University of Tennessee Health Science Center, Memphis, TN 38163 , USA

2Department of Dermatology, University of Alabama at Birmingham, Birmingham, AL 35294, USA

${ }^{3}$ Department of Tumor Pathology \& Pathomorphology, Oncology Center - Prof. Franciszek Łukaszczyk Memorial Hospital, The Ludwik Rydygier Collegium Medicum in Bydgoszcz, Nicolaus Copernicus University in Torun, Bydgoszcz, Poland

${ }^{4}$ School of Chemistry \& Biochemistry, the University of Western Australia, Crawley, Australia

*Author for correspondence: Tel.: +1 901448 3741; Fax: +1 901448 6979; aslominski@uthsc.edu

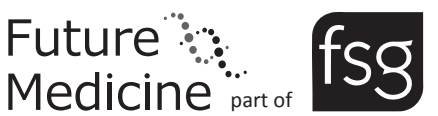

\section{KEYWORDS}

- adjuvant therapy $\bullet$ CYP11A1

- CYP27B1 - melanoma

- metabolism • vitamin D • vitamin

D receptor 
“There is accumulating evidence that melanoma development may be facilitated by vitamin $D$ deficiency and/or defects in the functioning of the vitamin D receptor."
Source \& activation of vitamin D

Exposure of human skin to ultraviolet (UV) wavelengths of solar radiation, including UVA $(\lambda=320-400 \mathrm{~nm})$ and UVB $(\lambda=280-320 \mathrm{~nm})$, has cytotoxic and mutagenic effects, and induces damage at the subcellular, cellular and tissue levels leading to a variety of skin pathologies including, but not limited to, epidermal skin cancers and melanoma [11,12]. In addition, UVB also has a positive effect on systemic homeostasis through induction of vitamin D3 (D3) formation in the skin [6], which after transport to the liver undergoes hydroxylation to 25-hydroxyvitamin $\mathrm{D} 3(25(\mathrm{OH}) \mathrm{D} 3)$. This serves as a prohormone that is activated to $1,25(\mathrm{OH})_{2} \mathrm{D} 3$ in the kidney or peripheral tissues that express the required enzyme, CYP27B1 [6]. The epidermis can also activate D3 locally through the same sequence of reactions: $\mathrm{D} \rightarrow 25(\mathrm{OH}) \mathrm{D} \rightarrow$ $1,25(\mathrm{OH})_{2} \mathrm{D}[7]$. Although the vast majority of systemic $\mathrm{D} 3$ derives from the skin, an additional source can be from dietary supplementation [6].

Over the last decade, we have identified novel pathways of vitamin D metabolism, initiated by the action of CYP11A1, that produce a number of hydroxy derivatives as follows: $\mathrm{D} 3 \rightarrow 20(\mathrm{OH}) \mathrm{D} 3 \rightarrow 20,23(\mathrm{OH})_{2} \mathrm{D} 3$ $\rightarrow 17,20,23(\mathrm{OH})_{3} \mathrm{D} 3$ and $\mathrm{D} 2 \rightarrow 20 S(\mathrm{OH}) \mathrm{D} 2$ $\rightarrow 17,20(\mathrm{OH})_{2} \mathrm{D} 2 \rightarrow 17,20,24(\mathrm{OH})_{3} \mathrm{D} 2[8,10]$. Our studies indicate that these pathways operate in vivo with CYP27B1 being capable of hydroxylating some of the products in the $1 \alpha$-position $[8,9]$. In tissues with high expression of CYP11A1, $20(\mathrm{OH}) \mathrm{D} 3$ is the major metabolite and is more abundant than 25(OH)D3 [8]. Similarly, tissues expressing low levels of CYP11A1 such as skin can also produce $20(\mathrm{OH}) \mathrm{D} 3$ and its hydroxy derivatives $[8,9]$. The initial and major metabolite of the pathway, $20(\mathrm{OH}) \mathrm{D} 3$, is detectable in human serum and epidermal keratinocytes [8].

\section{Defects in vitamin $\mathrm{D}$ signaling are associated with melanomagenesis \& melanoma progression}

There is accumulating evidence that melanoma development may be facilitated by vitamin $\mathrm{D}$ deficiency and/or defects in the functioning of the vitamin D receptor (VDR) [13-17]. Specifically, VDR polymorphisms are associated with an increased risk of developing melanoma (reviewed in [13]). Also, serum levels of $25(\mathrm{OH}) \mathrm{D} 3$ decrease with progression of metastatic melanoma [14]. Importantly, a negative correlation between melanoma progression and
VDR expression was shown with there being a remarkable decrease in VDR immunoreactivity in nuclei of melanoma cells at vertical versus radial growth phases, with metastatic melanomas showing the lowest receptor expression $[15,17]$. Also, the presence of ulceration and the lack of nonbrisk tumor infiltrating lymphocytes were accompanied by significantly lower VDR expression [17]. Furthermore, lack of or low expression of the VDR in primary melanomas and metastases was related to shorter overall patients' survival [15,17].

Similar observations were made concerning CYP27B1 [16], with expression being significantly lower in melanomas at the vertical growth phase and in metastatic melanomas compared with melanocytic nevi and melanomas at the radial growth phase. Expression was also significantly lower in more advanced stages (Clark's levels III-V, and Breslow's thickness $\geq 2.1 \mathrm{~mm}$ ) [16]. In addition, there was an inverse correlation between CYP27B1 and Ki-67 expression [16]. Furthermore, CYP27B1 expression was reduced in primary melanomas that developed metastases compared with nonmetastasizing melanomas. Importantly, the lack of or low expression of CYP27B1 was associated with shorter overall survival and disease-free survival time.

The above information indicates that defects in VDR signaling due to reduced receptor expression, low levels of $25(\mathrm{OH}) \mathrm{D} 3$ or its reduced activation (1 $\alpha$-hydroxylation) by CYP27B1 in the tumor environment, can be linked to melanomagenesis and tumor progression leading to shorter overall survival and disease-free survival time [16].

\section{Vitamin D \& growth of melanoma in} experimental models

Active forms of vitamin $\mathrm{D}$, including calcitriol $\left(1,25(\mathrm{OH})_{2} \mathrm{D} 3\right)$ and its derivatives, have been proven to display potent anticancer activity $[6,7,11]$ ) with the inhibition of melanoma growth (reviewed in $[13,18])$. Unfortunately, the hypercalcemic effect of high levels of $1,25(\mathrm{OH})_{2} \mathrm{D} 3$ attenuates the use of pharmacological doses for cancer therapy. However, its precursor, vitamin D3, has been successfully used at high doses $(50,000$ units per week or 10,000 units per day) in adjuvant treatment of autoimmune diseases (reviewed in [13]). Thus, vitamin D3/2 can potentially be used in the therapy of melanoma at relatively high doses providing patients are monitored for changes in serum calcium levels to prevent hypercalcemia. 
Importantly, we have discovered a new pathway of vitamin $\mathrm{D}$ metabolism initiated by CYP11A1, which produces novel analogs including 20-hydroxyvitamin D3/2 (20(OH) $\mathrm{D} 3 / 2)$. These show strong antileukemia and anticancer activities in vitro, while being noncalcemic and nontoxic in vivo $[10,19]$. We have already tested the in vitro activity of the major product of this pathway, $20(\mathrm{OH}) \mathrm{D} 3 / 2$ and its metabolites, and found that they show antiproliferative activity against cultured melanoma cells, acting through the VDR $[10,18,19] .20(\mathrm{OH})$ $\mathrm{D} 3 / 2$ and $20,23(\mathrm{OH})_{2} \mathrm{D} 3$ are also nontoxic in vivo at pharmacologically relevant doses [10], and are produced in vivo [8], defining them as an endogenous/natural products. These novel secosteroids demonstrate biological potency, equal or higher than that of $1,25(\mathrm{OH})_{2} \mathrm{D} 3$, with antiproliferative, prodifferentiation and anti-inflammatory activities on epidermal keratinocytes, melanocytes, melanoma cells and dermal fibroblasts. Importantly, $20(\mathrm{OH})$ D3 is noncalcemic and nontoxic at the highest pharmacological doses tested in rats $(3 \mu \mathrm{g} / \mathrm{kg})$ and in mice $(30-60 \mu \mathrm{g} / \mathrm{kg})$, which are up to 100 -fold higher than doses of $1,25(\mathrm{OH})_{2} \mathrm{D} 3$ or its precursor, $25(\mathrm{OH}) \mathrm{D} 3$, that are toxic [10]. Thus, we have identified a family of novel secosteroids that are excellent candidates for treatment of melanoma, since they can be used at very high doses that are nontoxic in animal models. The mechanism of the action of these secosteroids involves activation of the VDR [10,19] and/or action on alternative receptors including retinoic acid orphan receptor $\alpha$ and $\gamma[20]$.

\section{Vitamin D as a perfect adjuvant in therapy} of melanoma

Since vitamin D has anticancer and protective properties (reviewed in $[6,7,11,13]$ ), it can be used as an adjuvant during any type of therapy for stage III or IV melanoma. This proposal is substantiated by evidence showing that defects in vitamin D signaling are associated with melanomagenesis and its progression and that the majority of patients with advanced melanoma are vitamin $\mathrm{D}$ deficient or insufficient [13-17]. Furthermore, we propose the use of high doses of vitamin D3 (50,000 units per week or 10,000 units per day) for patients at disease stages I and II that have low levels of $25(\mathrm{OH})$ D3 $(<30 \mathrm{ng} / \mathrm{ml})$ as a prophylactic agent against recurrent or progressing disease. In this scenario, once serum levels of $25(\mathrm{OH}) \mathrm{D} 3$ reached $50-100 \mathrm{ng} / \mathrm{ml}$, the daily dose of vitamin D would be decreased to 4000-600 units per day, with the precise dose defined by serum calcium levels. This strategy could benefit melanoma patients at risk of developing metastatic disease (mitotically active ulcerated melanomas at the vertical growth phase that are relatively thick or with a positive sentinel lymph node biopsy).

\section{Conclusion \& future perspective}

Based on the antitumor properties of vitamin D and the link between defects in vitamin D signaling and melanoma development, we propose the use of high doses of vitamin $\mathrm{D}$, accompanied by monitoring of serum $25(\mathrm{OH}) \mathrm{D} 3 / 2$ and calcium levels, as an adjuvant or prophylactic strategy for disease stages III and IV or stages I and II, respectively. Preclinical testing of novel vitamin $\mathrm{D}$ analogs that are noncalcemic and can serve as natural products is mandatory to develop alternative or complementary strategies to treat this devastating disease.

Financial \& competing interests disclosure

Writing of this paper was supported in part by NIH grants 2R01AR052190, R21 AR066505-01A1 and 1R01AR056666 $01 A 2$ to AT Slominski. The authors have no other relevant affiliations or financial involvement with any organization or entity with a financial interest in or financial conflict with the subject matter or materials discussed in the manuscript apart from those disclosed.

No writing assistance was utilized in the production of this manuscript.
“6 potentially be used in the therapy of melanoma at relatively high doses providing patients are monitored for changes in serum calcium levels..."

\section{References}

1 Lo JA, Fisher DE. The melanoma revolution: from UV carcinogenesis to a new era in therapeutics. Science 346(6212), 945-949 (2014)

2 Schadendorf D, Hauschild A. Melanoma in 2013: melanoma-the run of success continues. Nat. Rev. Clin. Oncol. 11(2), 75-76 (2014).
3 Shah DJ, Dronca RS. Latest advances in chemotherapeutic, targeted, and immune approaches in the treatment of metastatic melanoma. Mayo Clin. Proc. 89(4), 504-519 (2014).

4 Kwong LN, Davies MA. Targeted therapy for melanoma: rational combinatorial approaches. Oncogene 33(1), 1-9 (2014).
5 Slominski AT, Carlson JA. Melanoma resistance: a bright future for academicians and a challenge for patient advocates. Mayo Clin. Proc. 89(4), 429-433 (2014).

6 Holick MF. Vitamin D deficiency. N. Engl. J. Med. 357(3), 266-281 (2007).

7 Bikle DD, Elalieh H, Welsh J, Oh D, Cleaver J, Teichert A. Protective role of vitamin $\mathrm{D}$ signaling in skin cancer 
formation. J. Steroid Biochem. Mol. Biol. 136, 271-279 (2013).

8 Slominski AT, Kim TK, Shehabi HZ et al. In vivo evidence for a novel pathway of vitamin $\mathrm{D}(3)$ metabolism initiated by P450scc and modified by CYP27B1. FASEB J. 26(9), 3901-3915 (2012).

9 Slominski AT, Kim TK, Shehabi HZ et al. In vivo production of novel vitamin D2 hydroxy-derivatives by human placentas, epidermal keratinocytes, Caco- 2 colon cells and the adrenal gland. Mol. Cell Endocrinol. 383(1-2), 181-192 (2014).

10 Slominski AT, Kim TK, Li W, Yi AK, Postlethwaite A, Tuckey RC. The role of CYP11A1 in the production of vitamin D metabolites and their role in the regulation of epidermal functions. J. Steroid Biochem. Mol. Biol. 144(Part A), 28-39 (2014).

11 Slominski AT, Zmijewski MA, Semak I et al. Cytochromes p 450 and skin cancer: role of local endocrine pathways. Anticancer Agents Med. Chem. 14(1), 77-96 (2014).

12 Slominski AT, Zmijewski MA, Skobowiat C, Zbytek B, Slominski RM, Steketee JD.
Sensing the environment: regulation of local and global homeostasis by the skin's neuroendocrine system. Adv. Anat. Embryol. Cell Biol. 212, 1-115 (2012).

13 Szyszka P, Zmijewski MA, Slominski AT. New vitamin $D$ analogs as potential therapeutics in melanoma. Expert Rev. Anticancer Ther. 12(5), 585-599 (2012).

14 Newton-Bishop JA, Davies JR, Latheef F et al. 25-hydroxyvitamin D2/D3 levels and factors associated with systemic inflammation and melanoma survival in the Leeds Melanoma Cohort. Int. J. Cancer doi:10.1002/ijc.29334. (2014) (Epub ahead of print).

15 Brozyna AA, Jozwicki W, Janjetovic Z, Slominski AT. Expression of vitamin D receptor decreases during progression of pigmented skin lesions. Hum. Pathol. 42(5), 618-631 (2011).

16 Brozyna AA, Jozwicki W, Janjetovic Z, Slominski AT. Expression of the vitamin D-activating enzyme 1alpha-hydroxylase (CYP27B1) decreases during melanoma progression. Hum. Pathol. 44(3), 374-387 (2013).
17 Brozyna AA, Jozwicki W, Slominski AT. Decreased VDR expression in cutaneous melanomas as marker of tumor progression: new data and analyses. Anticancer Res. 34(6), 2735-2743 (2014).

18 Slominski A, Janjetovic Z, Kim T-K et al. Novel vitamin $\mathrm{D}$ hydroxyderivatives inhibit melanoma growth and show differential effects on normal melanocytes. Anticancer Res. 32(9), 3733-3742 (2012).

19 Slominski AT, Kim TK, Janjetovic Z et al. 20-Hydroxyvitamin D2 is a noncalcemic analog of vitamin $\mathrm{D}$ with potent antiproliferative and prodifferentiation activities in normal and malignant cells. Am. J. Physiol. Cell Physiol. 300(3), C526-C541 (2011).

20 Slominski AT, Kim TK, Takeda Y et al. ROR alpha and ROR gamma are expressed in human skin and serve as receptors for endogenously produced noncalcemic 20-hydroxy- and 20,23-dihydroxyvitamin D FASEB J. 28(7), 2775-2789 (2014). 